\title{
STABILIZED OPTICAL FIBER LINKS FOR THE XFEL
}

\author{
Axel Winter, Universität Hamburg, Germany \\ Holger Schlarb, Bernhard Schmidt, DESY, Hamburg, Germany \\ F. Ömer Ilday, Jung-Won Kim, Jeff Chen, Felix J. Grawert, Franz X. Kärtner, \\ Massachusetts Institute of Technology, Cambridge, MA, USA
}

\begin{abstract}
The timing synchronization scheme for the European $\mathrm{X}$-Ray free electron laser facility (XFEL) is based on the generation and distribution of sub-picosecond laser pulses with actively stabilized repetition rate which are used to synchronize local RF oscillators. An integral part of the scheme is the distribution of the optical pulse stream to parts of the facility via optical fiber links. The optical path length of the fiber has to be stabilized against short-term and long-term timing jitter due to environmental effects, such as temperature drifts and acoustic vibrations, to better than $10 \mathrm{fs}$ for distances ranging from tens of meters to several kilometers. In this paper, we present first experimental results toward signal transmission through a $50 \mathrm{~m}$-long fiber link with femtosecond stability.
\end{abstract}

\section{INTRODUCTION}

One of the key challenges for the fourth generation light sources such as the XFEL will be to implement a timing synchronization system that allows the full exploitation of the potentially $10 \mathrm{fs}-$ long x-ray pulses for time-resolved studies. For this purpose, synchronization of various RF frequencies with femtosecond precision must be achieved between the critical components (injector, booster section, bunch compressors and experimental area) to ensure that the electron beam enters the undulator with timing jitter comparable to the pulse duration. These challenging requirements appears to be beyond the capability of traditional RF synchronization systems, based on temperature stabilized coaxial cables.

We have proposed an optical synchronization system to meet this challenge [1]: A train of sub-picosecond pulses of light generated from a mode-locked laser with very low timing jitter is distributed over actively length-stabilized optical fiber links to an arbitrary number of remote locations. At the remote locations, (i) a fraction of the incoming pulse is reflected back through the same fiber link to its point of origin to monitor synchronization, and (ii) lowlevel RF signal is extracted with high fidelity.

A critical component of this scheme is the fiber link, which must be stabilized against fluctuations in the refractive index of the fiber (due to temperature variations and acoustical noise) in order to maintain a fixed time of flight between two points. We expect the leading contribution to length variations to be thermal and mechani- cal effects, which occur at frequencies much less than 1 MHz. A rough estimate of these length variations can be obtained, given the thermal expansion coefficient of fiber $\left(\sim 2 \times 10^{-7} /{ }^{\circ} \mathrm{C}\right)$ [2]. In eventual system implementation, the fiber link can be maintained at a constant temperature within $1-2^{\circ} \mathrm{C}$ with a temperature stabilized fiber link. Such fiber is commercially available and has been deployed at similar facilities. Even then, the corresponding length variations in a $1 \mathrm{~km}$-long fiber link will be approximately $200 \mu \mathrm{m}$, corresponding to excess timing jitter of $\sim 1$ ps; clearly active stabilization is required.

\section{EXPERIMENTAL SETUP AND RESULTS}

We have constructed a miniature fiber link to test the feasibility of our approach. The experimental setup comprises of a mode-locked Yb-doped fiber laser (YDFL) [3], a single-mode fiber link with a total span of $50 \mathrm{~m}$, and optical cross-correlation between pulses transmitted through the fiber and pulses directly from the laser (Figure 1). In this experiment, the "remote station" to be synchronized is actually adjacent to the laser. Hence, for the sake of simplicity, the pulses are used directly out of the fiber link, instead of reflecting them back. We note that, although a YDFL with a central wavelength of $1030 \mathrm{~nm}$ is employed in this study, the results can be readily adopted to an Erdoped fiber laser operating at the telecommunication wavelength of $1550 \mathrm{~nm}$.

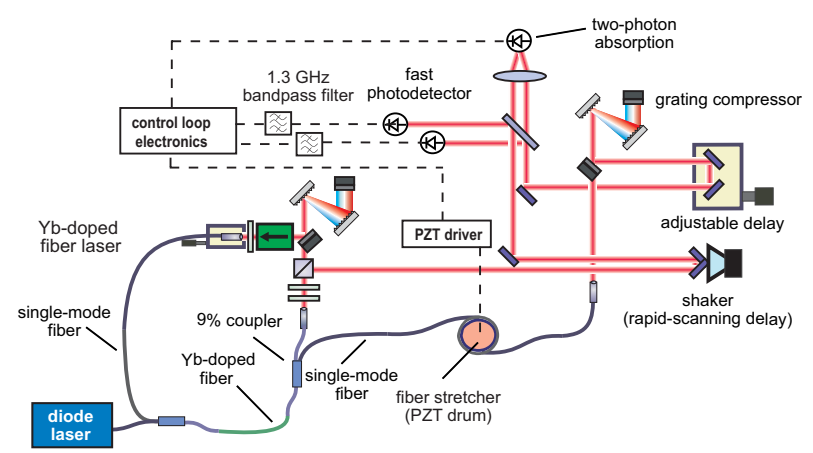

Figure 1: Schematic of the experimental setup.

The YDFL is configured to produce pulses compressible down to 70 fs with $2 \mathrm{~nJ}$ energy content, implementing stretched-pulse operation [4]. The optical spectrum is centered at $1030 \mathrm{~nm}$ (inset of Figure 5). The repetition rate of 
the pulse train is $45 \mathrm{MHz}$. Mode-locking is initiated and stabilized with nonlinear polarization evolution within the fiber, acting as an artificial saturable absorber. The laser has two output ports, a fiber coupler and a polarization rejection port.

Time of flight through the fiber link can be varied using several techniques, including changing the total optical path length via a motorized delay stage, or using an inline fiber stretcher. We adopt the latter method, due to its high modulation bandwidth (up to $\sim 40 \mathrm{kHz}$ ). The typical amount of path length variation which has to be compensated can be estimated from the thermally induced length variations to be $\sim 50 \mu \mathrm{m}$, assuming room temperature fluctuations of $\sim 5^{\circ} \mathrm{C}$. The fiber coupler directly seeds the fiber transmission link. About $40 \mathrm{~m}$ of the transmission fiber is wrapped tightly around a drum with a diameter of $\sim 10 \mathrm{~cm}$, which is stretched with piezo crystals. This fiber stretcher provides path length variations of $3.5 \mu \mathrm{m} / \mathrm{V}$. Group velocity dispersion of a medium (i.e., different frequencies experiencing different speeds of propagation) causes short pulses to broaden rapidly. The estimated dispersion of the fibers comprising the link is $+24 \mathrm{fs}^{2} / \mathrm{mm}$. Thus, upon transmission through link, the pulses are broadened to $\sim 42$ ps. A standard grating compressor compensates the dispersive broadening. The compressed pulses are characterized using intensity autocorrelation. However, the grating compressor allows compensation for only second-order dispersion. Residual higher-order dispersion limits the compressed pulse duration to approximately $250 \mathrm{fs}$.

For fiber links that will span large distances, the variations in optical path length will be significant. A robust approach necessitates being able to address both large displacements and to achieve femtosecond-level precision. An error signal can be obtained both from RF techniques and optical cross-correlation. Extremely high resolution can be obtained using optical cross-correlation between the transmitted pulse and a pulse directly from the master oscillator; the resolution is limited to (a small) fraction of the pulse width. However, a corollary of this statement is that the maximum displacement that can be measured is order of one pulse width (beyond that, there is no overlap between the pulses, hence no cross-correlation signal is generated). Therefore, we adopt the approach of using RF-locking for coarse overlap and optical cross-correlation via two-photon absorption for extra-fine resolution (Figure 2).

A fraction of the pulses from the fiber link and those directly from the laser are redirected with a beamsplitter onto high-bandwidth photodetectors. In order to obtain a high-quality lock in the order of $100 \mathrm{fs}$, a high harmonic is selected with a suitable bandpass filter. After amplification, both signals are fed to a phase detector, which converts the phase difference of the two input signals to an error voltage. The resulting error signal is then amplified by the loop filter, which is a proportional-integral controller with a lowpass filter. The proportional and integral parts are in parallel, so the advantages of an integrator at low frequencies can be obtained without compromising the phase margin at

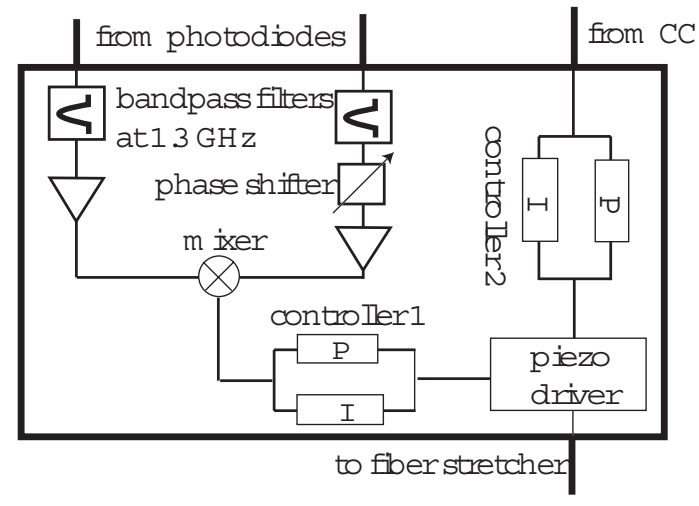

Figure 2: Schematic of the electronic feedback circuit.

higher frequencies. After being amplified to higher voltage levels to suit the piezo-based fiber stretcher, the loop is closed by feeding the signal to the stretcher.

Likewise, for the fine resolution feedback loop, the cross correlation signal is filtered and amplified by a low-noise amplifier (Stanford Research SR650). The signal then traverses a loop filter, and connected to the stretcher upon amplification by the piezo driver. In general, the locking points of both feedback will not be equal. This is rectified with a phase shifter introduced into one arm of the RF feedback, ensuring overlap between the locking points of the cross correlation signal and the RF feedback.

The fiber stretcher adjusts the length of the fiber link in proportion to the applied voltage $(3.5 \mu \mathrm{m} / \mathrm{V})$. Its transfer function can be expressed as a proportional element. For overall stability, mechanical resonances of the piezo crystal inside the fiber stretcher need to be considered, which ultimately limit the gain. We model the mechanical resonance (measured to be at $18 \mathrm{kHz}$ ) with a harmonic oscillator, which limits the achievable unity gain to around 4 $\mathrm{kHz}$. If higher unity gain is desired, a combination of 2 fiber stretchers, one with higher stroke to compensate for slow temperature drifts and another with short stroke but higher resonance frequency can be used. The simulated open loop transfer function is shown in Figure 3.

The power spectral density of the error signal of the phase detector is illustrated in Figure 4 for the free-running and the stabilized fiber link. The measurement is limited by the noise floor of the vector signal analyzer for frequencies above $10 \mathrm{kHz}$. When the feedback loop is open, the beam traveling through the fiber has an added timing jitter of 5.5 fs rms within the frequency band of $10 \mathrm{mHz}-10 \mathrm{kHz}$. The stabilization scheme successfully suppresses the perturbations, particularly in the low-frequency range. The added timing jitter is down to $2.9 \mathrm{fs}$ rms within the frequency band of $10 \mathrm{mHz}$ to $10 \mathrm{kHz}$.

The cross-correlation of the compressed pulses from the fiber link and the unaltered pulses directly from the polarization port of the YDFL is obtained using two-photon absorption in a photodetector as the nonlinear coincidence signal. This method is simpler to implement, lower-cost 


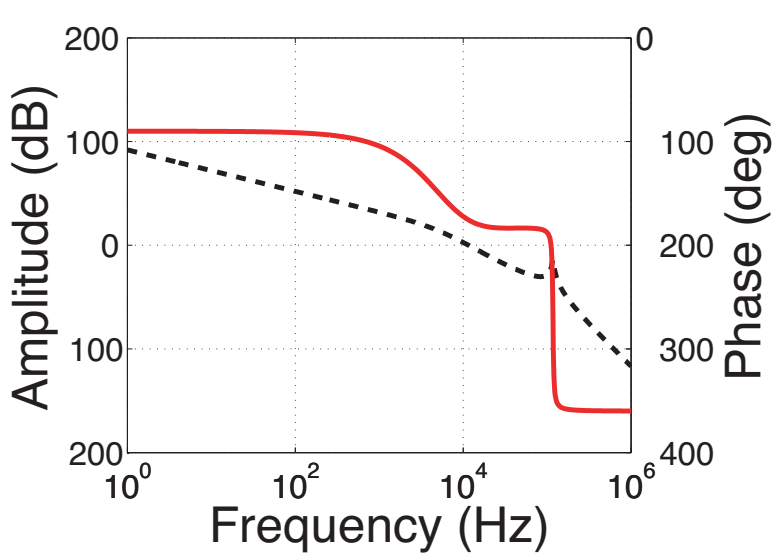

Figure 3: Simulated open loop transfer function: amplitude (black, dashed line) and phase (red. solid line).

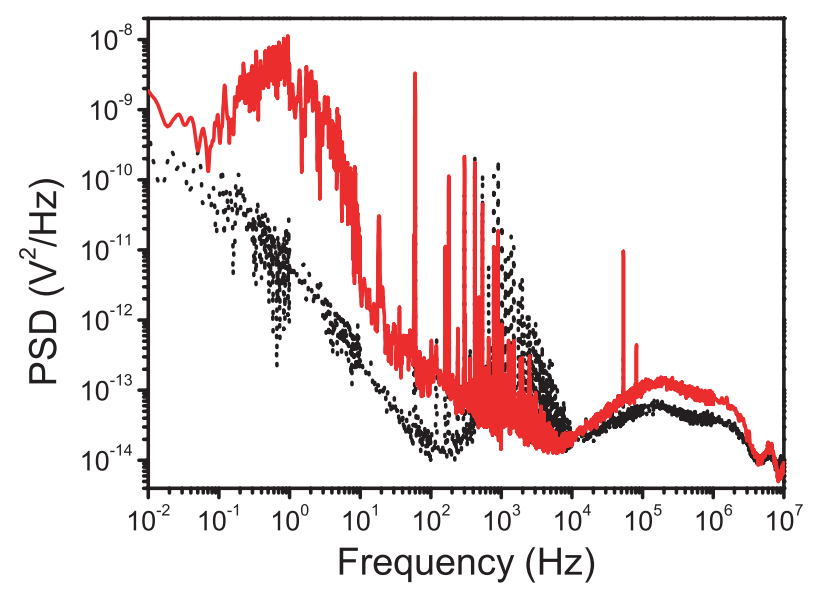

Figure 4: Measured power spectral density of the in-loop noise for the stabilized (red, solid line) and the free-running (black, dashed line) fiber link.

and polarization-insensitive, in comparison to the widely used method of second-harmonic generation in a nonlinear crystal. Coarse temporal overlap of the pulses from the fiber link and directly from the laser is achieved using a long-range delay stage. The cross-correlation trace is then generated by rapidly scanning the delay between the two pulses with a short-range shaker, which is simply an audio speaker (Figure 5). In the absence of any variations in the length of the fiber link, the cross-correlation trace corresponds to a convolution of the two pulses. The effect of the length fluctuations is to shift in time and broaden the crosscorrelation signal. In fact, the effect is readily observable when the room temperature is varied by turning on or off the air conditioner. We expect that stabilization through the use of the optical cross-correlation will enable reduction of the added timing jitter well below 20 fs up to the Nyquist frequency. Work is in progress for the implementation of the second, fine-resolution feedback loop based on the optical cross-correlation.

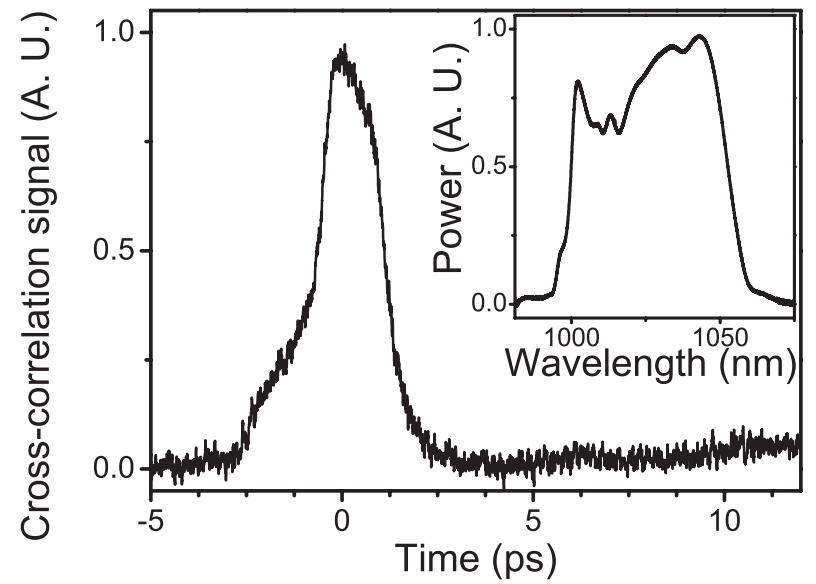

Figure 5: Measured optical cross-correlation of the freerunning fiber link, using two-photon absorption. Inset: Optical spectrum of the mode-locked YDFL.

\section{CONCLUSION AND OUTLOOK}

In conclusion, the proposed optical timing synchronization scheme is emerging as an attractive solution for next generation light sources. The ultimate utility of such this scheme rests upon the demonstration of several key steps. In addition to the development of ultra-low timing jitter fiber lasers as master oscillators, timing-stabilized transmission of the pulse train over long fiber links must be demonstrated. In this work, we have obtained active stabilization for sub-picosecond pulses over a $50 \mathrm{~m}$ of singlemode fiber link using RF-locking techniques. The added timing jitter of $5.5 \mathrm{fs}$ is reduced to $2.9 \mathrm{fs}$ with stabilization $(10 \mathrm{mHz}-10 \mathrm{kHz})$. For ultra-precise locking, optical cross-correlation will be utilized, allowing a resolution of a small fraction of the pulse duration. The error signal based on cross-correlation has been demonstrated. Implementation of the additional, ultra-precise stabilization loop based on cross-correlation is under way. Finally, these proof-ofprinciple experiments can be readily generalized to greater length spans.

\section{REFERENCES}

[1] A. Winter, P. Schmüser, H. Schlarb, F. Ö. Ilday, J. Kim, J. Chen, and F. X. Kärtner, "Phase Noise Characteristics of Fiber Lasers as Potential Ultra-Stable Master Oscillators," in proceedings, PAC 2005, Knoxville, TN, USA.

[2] C. E. Lee, H. F. Taylor, A. M. Markus, and E. Udd, "Opticalfiber Fabry - Perot embedded sensor," Opt. Lett. 14, 12251227 (1989).

[3] F. Ö. Ilday, J. R. Buckley, H. Lim, and F. W. Wise, "Generation of 50-fs, 5-nJ pulses at $1.03 \mu \mathrm{m}$ from a wave-breakingfree fiber laser," Opt. Lett. 28, 1365-1367 (2003).

[4] K. Tamura, E. P. Ippen, H. A. Haus, and L. E. Nelson, "77fs pulse generation from a stretched-pulse mode-locked allfiber ring laser," Opt. Lett. 18, 1080-1082 (1993). 